\title{
Implant R100 Predicts Rectal Bleeding in Prostate Cancer Patients Treated with IG-IMRT to 45 Gy and Pd-103 Implant
}

\author{
Matthew Packard, ${ }^{1}$ Vladimir Valakh, ${ }^{2}$ and Russell Fuhrer ${ }^{1}$ \\ ${ }^{1}$ Department of Radiation Oncology, Allegheny General Hospital, 320 East North Avenue, Pittsburgh, PA 15212-9986, USA \\ ${ }^{2}$ Department of Radiation Oncology, Temple University Hospital, 3401 N Broad Street, Philadelphia, PA 19140, USA
}

Correspondence should be addressed to Matthew Packard; packardmw@gmail.com

Received 26 September 2013; Revised 29 November 2013; Accepted 13 December 2013; Published 4 February 2014

Academic Editor: Andreas Meyer

Copyright (C) 2014 Matthew Packard et al. This is an open access article distributed under the Creative Commons Attribution License, which permits unrestricted use, distribution, and reproduction in any medium, provided the original work is properly cited.

\begin{abstract}
Purpose. To define factors associated with rectal bleeding in patients treated with IG-IMRT followed by Pd-103 seed implant. Methods and Materials. We retrospectively reviewed 61 prostate adenocarcinoma patients from 2002 to 2008. The majority (85.2\%) were of NCCN intermediate risk category. All received IG-IMRT to the prostate and seminal vesicles followed by Pd-103 implant delivering a mean D90 of 100.7 Gy. Six patients received 45 Gy to the pelvic nodes and 10 received androgen deprivation. Results. Ten patients (16.4\%) developed rectal bleeding: 4 were CTCAE v.3 grade 1, 5 were grade 2, and 1 was grade 3 . By univariate analysis, age, stage, Gleason sum, PSA, hormonal therapy, pelvic radiation, postoperative prostate volume, D9, V100, individual source activity, total implanted activity per $\mathrm{cm}^{3}$, and duration of interval before implant did not impact rectal bleeding. Implant R100 was higher in patients with rectal bleeding: on average, 0.885 versus $0.396 \mathrm{~cm}^{3}, P=.02$, odds ratio of 2.26 per $.5 \mathrm{~cm}^{3}(95 \% \mathrm{CI}, 1.16-4.82)$. A trend for significance was seen for prostate V200 and total implanted activity. Conclusion. Higher implant R100 was associated with development of rectal bleeding in patients receiving IG-IMRT to 45 Gy followed by Pd-103 implant. Minimizing implant R100 may reduce the rate of rectal bleeding in similar patients.
\end{abstract}

\section{Introduction}

Prostate cancer is the most common cancer in American men and their second leading cause of cancer death [1]. The combination of brachytherapy and external beam radiotherapy (EBRT) is a common treatment regimen for patients with intermediate and high-risk prostate cancer. This combination allows higher doses to be delivered safely to the prostate than is possible with EBRT alone. Previous researchers have shown that increasing biologically equivalent dose (BED) delivered, whether from brachytherapy alone or from the combination of EBRT plus brachytherapy, results in a strong dose-response relationship with regard to both freedom from biochemical failure and rates of posttreatment negative biopsy [2]. The combination of EBRT and brachytherapy also increases convenience to the patient by shortening treatment time from 9 to 5 weeks compared to full-dose EBRT.

External beam radiotherapy can be delivered using a variety of techniques and, in recent years, many centers have transitioned from 3D conformal radiotherapy to intensity modulated radiation therapy (IMRT) for the delivery of external beam prostatic radiotherapy. IMRT technique allows higher doses to be delivered to targets at risk while delivering decreased doses to surrounding normal structures, such as bladder, rectum, small bowel, and femoral heads [35]. Additionally, many centers have begun to utilize daily image guidance to ensure accurate daily target localization in conjunction with IMRT (IG-IMRT) [6]. Image guidance attempts to account for interfractional prostatic movement, thus minimizing geographic misses and potentially allowing the use of smaller planning target volume (PTV) margins and dose escalation.

Authors from this institution have previously published data assessing GU and GI toxicity in a cohort of prostate cancer patients treated with the combination of IG-IMRT and seed implant and found approximately $15 \%$ of patients experienced at least one episode of GI bleeding which could be attributable to radiation [7]. Other researchers have shown 
that increasing rectal volumes receiving high radiation dose correlated with rectal bleeding risk in patients receiving brachytherapy alone $[8,9]$, EBRT combined with reduced dose Pd-103 implant [10, 11], and EBRT combined with I-125 implant $[12,13]$. However, for a common treatment regimen (IG-IMRT to 45 Gy followed by Pd-103 boost), patient and treatment factors associated with rectal bleeding remain undefined. The purpose of this study was to attempt to identify patient and treatment parameters that correlated with rectal bleeding in patients treated with IG-IMRT to $45 \mathrm{~Gy}$ followed by Pd-103 brachytherapy at a single institution.

\section{Methods and Materials}

2.1. Patients. We identified sixty-one consecutive patients with clinically localized prostate adenocarcinoma who were treated with curative intent using the combination of IGIMRT to 45 Gy followed by Pd-103 implant boost at a single institution from 2002 to 2008 . Patients were identified using a prospectively maintained clinical database approved by the institution's internal review board. All patients had histologically proven adenocarcinoma of the prostate diagnosed by transrectal needle biopsy and slides were reviewed by a single pathologist. The presence of detectable regional and distal metastases was ruled out in patients at intermediate and high risk for extracapsular spread.

Patients were staged according to the American Joint Committee on Cancer clinical staging guidelines and included those with clinical stage T1c through T2c, with the majority being T1c $(n=34,55.7 \%)$ or T2a $(n=19,31.1 \%)$. The median pretreatment serum prostate specific antigen (PSA) level was $5.4 \mathrm{ng} / \mathrm{mL}$ (mean, $6.8 \mathrm{ng} / \mathrm{mL}$; range, $1.7-$ $18 \mathrm{ng} / \mathrm{mL}$ ). The majority of patients had a Gleason score of 7 (78.7\% of patients). Risk group for patients was as defined by the National Comprehensive Cancer Network (NCCN). The majority were intermediate risk $(n=52,85.2 \%)$. Median age was 68 years (range 51-76 years). Additional patient characteristics are listed in Table 1.

2.2. External Beam Radiation Therapy. All patients received daily external beam ( $\geq 6 \mathrm{MV}$ ) radiation to the prostate and proximal seminal vesicles to a dose of $45 \mathrm{~Gy}$ in 25 fractions at $1.8 \mathrm{~Gy} /$ fraction. The clinical target volume (CTV) included the prostate and proximal seminal vesicles with a PTV margin of $1 \mathrm{~cm}$ added in all directions except for the posterior margin, which was reduced to $8 \mathrm{~mm}$.

All patients received CT simulation using a Siemens Somatom Open CT scanner. IV contrast was administered to aid in delineating the interface between the bladder and prostatic tissue. CTV delineation and contouring were performed on $3 \mathrm{~mm}$ axial CT slices. Rectal volumes were created by outlining a solid structure including the rectal wall extending from at least $2 \mathrm{~cm}$ proximal and distal to the prostate CTV. IMRT plans were created using the CMS $\mathrm{XiO}$ treatment planning system (CMS, St. Louis, MO). As it is possible that patients planned for the combination of EBRT followed by brachytherapy will elect not to undergo the brachytherapy procedure or may not be able to undergo
TABLE 1: Patient characteristics.

\begin{tabular}{lcc}
\hline Characteristic & No. of patients $(n=61)$ & $\%$ of total \\
\hline Age $(\mathrm{yrs})$ & & \\
$\quad \leq 65$ & 22 & $36.1 \%$ \\
$\quad>65$ & 39 & $63.9 \%$ \\
Pretreatment PSA (ng/mL) & & \\
$\quad<10$ & 50 & $82.0 \%$ \\
$\geq 10$ & 11 & $18.0 \%$ \\
Gleason score & & \\
6 & 7 & $11.5 \%$ \\
7 & 48 & $78.7 \%$ \\
8 & 6 & $9.8 \%$ \\
Tumor stage & & \\
T1c & 34 & $55.7 \%$ \\
T2a & 19 & $31.1 \%$ \\
T2b & 6 & $9.8 \%$ \\
T2c & 2 & $3.3 \%$ \\
Risk group & & \\
Low & 3 & $4.9 \%$ \\
Intermediate & 52 & $85.2 \%$ \\
High & 6 & $9.8 \%$ \\
Androgen deprivation therapy & & \\
No & 50 & \\
Yes & 11 & \\
\hline
\end{tabular}

PSA: prostate specific antigen.

the procedure, EBRT plans were calculated and optimized to deliver $79.2 \mathrm{~Gy}$ and rescaled to $45 \mathrm{~Gy}$. Using the plan to deliver 79.2 Gy, rectal and bladder doses were limited to a V70 of less than $15 \mathrm{cc}$ and V65 less than 40\%, respectively. Androgen deprivation therapy using luteinizing hormonereleasing hormone agonist therapy (LH-RH) was used in 11 patients (18\%). Six patients also received simultaneous external irradiation of pelvic lymph nodes to $45 \mathrm{~Gy}$ using IMRT technique.

For the patients whose pelvic lymph nodes were irradiated, the pelvic CTV included the obturator, external iliac, proximal internal iliac, and common iliac node regions, identified using the vascular structures, up to the level of L5S1. A PTV margin of $1.2 \mathrm{~cm}$ was added in all directions.

Daily image guidance was utilized for all patients. B-mode acquisition and targeting ultrasound-based system (BAT) was used in 39 patients (63.9\%), fiducial system of intraprostatic gold marker seeds in 9 patients (14.7\%), megavoltage cone-beam CT (MV-CBCT) combined with fiducial marker seeds in 8 patients $(13.1 \%)$, and MV-CBCT alone in 5 patients (8.1\%).

2.3. Seed Implant. All patients received Pd-103 transperineal brachytherapy at a median of 22 days following completion of IMRT (range 7-85 days). Seed implant was prescribed to a D90 of $100 \mathrm{~Gy}$. Seeds were implanted using a Mick applicator with intraoperative real-time computer planning and transrectal ultrasound guidance. Brachytherapy treatment planning was performed using Variseed 9.1. The median 
individual seed activity was $1.8 \mathrm{U}$ (range 1.3-2.5 U). Median number of seeds implanted was 75 seeds (range 44-122 seeds). The median total implanted activity was $132.1 \mathrm{U}$ (range 79.6-178.6 U). Median delivered D90 was 100.7 Gy (range 78.6-146.6 Gy).

2.4. Dosimetric Parameters. Within 30 days, all patients underwent CT for dosimetry and implant quality assessment. One investigator constructed contours of the prostate and rectum on $3 \mathrm{~mm}$ axial CT slices of all patients. Using Variseed 9.1 software, the rectum was contoured as a solid structure based on the outer border of the rectal wall from $1 \mathrm{~cm}$ above the most superior slice containing identifiable seeds to $1 \mathrm{~cm}$ below the most inferior slice displaying identifiable seeds. A number of rectal DVH parameters based on the postoperative implant assessment were computed including mean rectal dose, rectal volume, prostate V150, prostate V200, and implant R100 (rectal volume receiving 100\% of prescribed implant dose).

2.5. Statistical Methods. Data analysis was performed using SPSS v.18 (International Business Machines, North Castle, NY, USA). Total incidence of rectal bleeding of any grade was described as a crude rate. Time from brachytherapy to development of rectal bleeding was not recorded, but all events occurred within 24 months of brachytherapy. For univariate analysis, Mann-Whitney $U$ test was used for continuous variables and chi-square test used for categorical variables. Independent variables with a trend towards statistically significant association with rectal bleeding were included in a logistic regression model. Significance level was set at $P=0.05$.

\section{Results}

Toxicity was graded using the CTCAE v.3 grading scale [14]. With a median followup of 28 mos (range 12-89 mos), 10 patients experienced rectal bleeding. Four developed grade 1 bleeding (self-limiting), 5 developed grade 2 bleeding (requiring medical management), and 1 developed grade 3 bleeding (requiring argon plasma coagulation). There were no instances of grade 4 or 5 rectal bleeding (Table 3 ).

By univariate analysis, age, stage, Gleason sum, serum PSA, hormonal therapy, pelvic node irradiation, postoperative prostate volume, prostate D90 and V100 (relative volume receiving $100 \%$ of prescribed implant dose), individual source activity, and duration of interval before implant were not associated with rectal bleeding of any grade (Table 2 ). The mean implant R100 was significantly higher in patients with rectal bleeding compared to those without bleeding $\left(0.885 \mathrm{~cm}^{3}\right.$ vs $\left.0.396 \mathrm{~cm}^{3}, P=0.021\right)$. A trend for significance was seen for prostate V200 $(P=0.07)$ and total implanted activity $(P=0.09)$.

Prostate V200 strongly correlated with implant R100 $(P<$ 0.001). Therefore only implant R100 and total implanted activity were included in the multivariate logistic regression model. The model was statistically a good fit $(P<0.05)$. By multivariate analysis, only implant R100 was associated with
TABLE 2: Univariate and multivariate analyses.

\begin{tabular}{lcc}
\hline Variable & $\begin{array}{c}\text { Univariate } \\
P \text { value }\end{array}$ & $\begin{array}{c}\text { Multivariate } \\
P \text { value }\end{array}$ \\
\hline R100, cc & 0.02 & 0.02 \\
Prostate V200, \% & 0.07 & \\
Total source activity, U & 0.09 & 0.31 \\
Follow-up duration, d & 0.12 & \\
Prostate V150, \% & 0.17 \\
Number of implanted seeds, $n$ & 0.20 \\
T stage & 0.21 \\
Prostate volume, cc & 0.28 \\
Risk group & 0.36 \\
PSA, $n$ & 0.40 \\
Hormonal therapy & 0.47 \\
Total activity to prostate volume ratio & 0.60 \\
Time between IG-IMRT and implant, d & 0.61 \\
Age, y & 0.71 \\
Individual source activity, mCi & 0.73 \\
Prostate V100, \% & 0.83 \\
Gleason score, $n$ & 0.89 \\
D90, Gy & 0.91 \\
Pelvic radiation & 0.99 \\
\hline
\end{tabular}

PSA: prostate specific antigen.

rectal bleeding $(P=0.02)$, with an odds ratio of 2.26 per $0.5 \mathrm{~cm}^{3}$ (95\% confidence intervals, $1.16-4.82$ ).

Mean implant R100 was $0.885 \mathrm{cc}$ for those with rectal bleeding versus $0.396 \mathrm{cc}$ for those without rectal bleeding. The most appropriate threshold implant R100 value which might predict rectal bleeding to test for significance was $0.6 \mathrm{cc}$. Five of 19 patients with an implant R100 greater than or equal to $0.6 \mathrm{cc}$ had rectal bleeding (0.26), versus $5 / 42$ patients with implant R100 less than $0.6 \mathrm{cc}(0.12)$. This was tested for statistical significance using a 2-sample $z$-test with a desired significance level of 0.05 . The threshold implant R100 of $0.6 \mathrm{cc}$ resulted in a $P$ value of 0.08 ; thus this threshold was not found to be statistically significant, most likely due to the relatively small sample sizes and low instances of rectal bleeding.

\section{Discussion}

Despite the use of this highly conformal approach to definitively treating intermediate and high-risk prostate cancer with radiotherapy, the combination of IG-IMRT and seed implant can be associated with complications including rectal bleeding. As initially described by Snyder et al. [9], studies examining the association between implant DVH parameters and postimplant toxicity are important to optimize planning techniques to minimize the likelihood and severity of such adverse effects. As mentioned in Section 1 authors from this institution previously reported that the combination of IGIMRT and Pd-103 prostate brachytherapy in patients with organ confined prostate cancer can be associated with rectal bleeding [7]. The current study more thoroughly examines 
TABLE 3: Individual D90 and implant R100 values.

\begin{tabular}{|c|c|c|c|c|c|c|c|}
\hline Patient no. & D90 (Gy) & Implant R100 (cc) & Rectal bleeding & Patient no. & D90 (Gy) & Implant R100 (cc) & Rectal bleeding \\
\hline 1 & 78.6 & 0.27 & None & 32 & 101.0 & 0.09 & None \\
\hline 2 & 85.5 & 0.16 & None & 33 & 101.7 & 0.52 & None \\
\hline 3 & 87.2 & 0.19 & None & 34 & 104.0 & 0.83 & None \\
\hline 4 & 89.2 & 0.30 & None & 35 & 104.1 & 0.63 & None \\
\hline 5 & 89.2 & 0.77 & Grade 2 & 36 & 104.6 & 0.18 & None \\
\hline 6 & 89.8 & 0.05 & None & 37 & 105.0 & 0.12 & None \\
\hline 7 & 90.3 & 0.05 & None & 38 & 107.0 & 0.24 & None \\
\hline 8 & 90.4 & 0.01 & None & 39 & 107.1 & 0.87 & None \\
\hline 9 & 91.2 & 0.00 & None & 40 & 107.2 & 0.37 & None \\
\hline 10 & 93.3 & 0.55 & None & 41 & 107.3 & 1.76 & None \\
\hline 11 & 93.5 & 0.04 & None & 42 & 107.6 & 0.06 & None \\
\hline 12 & 93.6 & 0.00 & None & 43 & 107.8 & 0.02 & None \\
\hline 13 & 94.7 & 0.41 & None & 44 & 108.8 & 0.80 & None \\
\hline 14 & 94.7 & 0.26 & None & 45 & 109.1 & 0.34 & None \\
\hline 15 & 94.9 & 0.09 & None & 46 & 111.5 & 0.73 & None \\
\hline 16 & 95.7 & 0.29 & None & 47 & 112.1 & 1.83 & Grade 1 \\
\hline 17 & 95.9 & 0.32 & None & 48 & 112.3 & 0.08 & None \\
\hline 18 & 96.8 & 0.95 & None & 49 & 113.2 & 0.44 & None \\
\hline 19 & 97.1 & 1.91 & Grade 1 & 50 & 114.3 & 0.06 & Grade 3 \\
\hline 20 & 97.4 & 1.05 & None & 51 & 114.8 & 0.00 & None \\
\hline 21 & 98.1 & 0.00 & None & 52 & 118.0 & 0.80 & None \\
\hline 22 & 98.2 & 0.27 & Grade 1 & 53 & 119.7 & 0.36 & None \\
\hline 23 & 98.3 & 1.02 & Grade 2 & 54 & 120.6 & 0.05 & None \\
\hline 24 & 99.0 & 0.30 & Grade 1 & 55 & 121.3 & 0.02 & None \\
\hline 25 & 99.1 & 0.58 & Grade 2 & 56 & 122.7 & 0.50 & None \\
\hline 26 & 99.7 & 0.23 & None & 57 & 123.6 & 0.86 & None \\
\hline 27 & 99.8 & 0.75 & None & 58 & 126.0 & 1.22 & None \\
\hline 28 & 100.0 & 0.24 & None & 59 & 127.2 & 0.89 & None \\
\hline 29 & 100.1 & 0.37 & Grade 2 & 60 & 133.0 & 1.74 & Grade 2 \\
\hline 30 & 100.3 & 0.04 & None & 61 & 146.6 & 1.05 & None \\
\hline 31 & 100.7 & 0.10 & None & & & & \\
\hline
\end{tabular}

R100: volume of rectum receiving 100\% of prescribed dose.

patients and disease and treatment parameters in an attempt to identify those which correlated with rectal bleeding. We found only one variable that statistically correlated with rectal bleeding, that being the volume of rectum receiving $100 \%$ of prescribed implant dose (R100). We were unable to identify a threshold implant R100, which, if exceeded, predicts rectal bleeding.

Our results corroborate those of a recently published study which also showed that implant R100 correlated with rectal bleeding [12]. That study showed that, in addition to implant R100, implant R150 also correlated with rectal bleeding, which was not analyzed in the current study. There were also important differences between that study and the current study. External beam radiotherapy (EBRT) preceded seed implant in our study, while seed implant was performed first in their cohort. Our rate of rectal bleeding (16.4\%) was somewhat higher than the rate reported in that study $(9.7 \%)$; however, they only looked at rectal bleeding of at least grade 2 . We included any grade rectal bleeding. If patients with grade one bleeding were excluded from our analysis, our rate of grade 2 or greater rectal bleeding is comparable at $9.8 \%$ (6/61 patients). Another difference is that the radioisotope used in our study was Pd-103, while their patients received seed implants using I-125. Although there are differences in the half-lives and half-value layers of the isotopes, our results suggest that the rate of rectal bleeding depends more upon the implant R100 than the choice of radioisotope. Finally, our cohort was treated with IG-IMRT and relatively small margins while theirs received $3 \mathrm{D}$ conformal radiotherapy with wider PTV margins without image guidance.

A subset analysis of a separate study also showed that implant R100 correlated with GI toxicity in patients receiving EBRT and seed implant [13]. In the subset of 48 patients who received both EBRT and seed implant, implant R100 was associated with grade 2 or greater GI toxicity. Additionally, implant dose to the hottest $0.1 \mathrm{cc}$ of rectum, implant dose to the hottest $1 \%$ of rectum, and implant dose to the hottest $3 \%$ of rectum were also associated with grade 2 or greater 
GI toxicity. These less commonly reported metrics were not evaluated in our study. As in the previously mentioned study, our study differed from theirs in that we used Pd-103 rather than I-125 as the radioisotope for the seed implant. Nonetheless, again the choice of isotope did not seem to negate the importance of implant R100, as they reported similar findings to our study.

Another study investigated factors predictive of rectal bleeding following EBRT and Pd-103 seed implant in a subset of patients treated in a large prospective clinical trial [11]. Patients were enrolled in this multi-institutional trial with a $2 \times 2$ design randomizing between 44 versus $20 \mathrm{~Gy}$ EBRT and 90 versus $115 \mathrm{~Gy}$ implant dose, resulting in a somewhat heterogeneous treatment pattern. A four-field box arrangement was used to treat the prostate and seminal vesicles with a $2 \mathrm{~cm}$ margin, reduced to $1 \mathrm{~cm}$ posteriorly. They found that rectal bleeding was related to implants R100, R200, and R300.

In Radiation Therapy Oncology Group P-0019, a prospective phase II study of the combination of EBRT without image guidance to $45 \mathrm{~Gy}$ followed by I- 125 implant, there was no grade 3 or greater rectal bleeding. They did not report rates of grade 1-2 rectal bleeding $[15,16]$. Another study, Radiation Therapy Oncology Group 0232, a phase III trial comparing the combination of EBRT and seed implant versus seed implant alone in selected intermediate risk patients, is closed to accrual with results pending.

Our study has several limitations. The median duration of followup was relatively short at 28 months and it is possible that toxicity rates will be higher in subjects followed up longer. Given the typical time course of radiotherapyrelated rectal bleeding, an actuarial analysis is the preferred method of assessing rates of rectal bleeding. However in this study, dates of rectal bleeding onset were not recorded; thus an actuarial analysis is not able to be performed and a comparison of crude rates was performed.

Another limitation is that we did not conduct a detailed DVH analysis of the external beam portion of the patient's combination therapy. Nonetheless, the EBRT rectal DVH criteria described above were met in all patients. Additionally, other implant rectal dose metrics such as implant R150, implant R200, and implant R300, some of which were analyzed in other studies but were not analyzed in this study, may also be useful to investigate their relationship to rectal bleeding. Finally, direct comparison of our rates of adverse events to other studies, some of which are prospective, multi-institutional trials, is not possible because of major differences in study design and implementation, number of patients, duration of followup, and differing toxicity scales used.

\section{Conclusion}

Several studies have shown that increasing rectal volumes receiving high dose from brachytherapy impact the risk of developing rectal bleeding in patients receiving the combination of 3D conformal or non-image-guided IMRT and seed implant as definitive treatment for prostate cancer. Ours is the first study to show that this relationship exists in a commonly employed modern regimen of IG-IMRT to $45 \mathrm{~Gy}$ in 25 fractions followed by Pd-103 seed implant. Calculating implant R100 and making an attempt to minimize it may reduce the rate of rectal bleeding in prostate cancer patients treated with IG-IMRT to $45 \mathrm{~Gy}$ followed by Pd-103 implant.

\section{Conflict of Interests}

The authors declare that there is no conflict of interests regarding the publication of this paper.

\section{Acknowledgments}

The authors thank Katsiaryna Mazurenka, M.A., for statistical consultation and the Western Pennsylvania Prostate Foundation, Pittsburgh, PA, for supporting the data collection.

\section{References}

[1] American Cancer Society, Cancer Facts and Figures 2010, American Cancer Society, Atlanta, Ga, USA, 2010.

[2] R. G. Stock, N. N. Stone, J. A. Cesaretti, and B. S. Rosenstein, "Biologically effective dose values for prostate brachytherapy: effects on PSA failure and posttreatment biopsy results," International Journal of Radiation Oncology, Biology, Physics, vol. 64, no. 2, pp. 527-533, 2006.

[3] M. J. Zelefsky, Z. Fuks, M. Hunt et al., "High-dose intensity modulated radiation therapy for prostate cancer: early toxicity and biochemical outcome in 772 patients," International Journal of Radiation Oncology, Biology, Physics, vol. 53, no. 5, pp. 11111116, 2002.

[4] O. Cahlon, M. J. Zelefsky, A. Shippy et al., "Ultra-high dose (86.4 Gy) IMRT for localized prostate cancer: toxicity and biochemical outcomes," International Journal of Radiation Oncology, Biology, Physics, vol. 71, no. 2, pp. 330-337, 2008.

[5] I. Latorzeff, J. Mazurier, C. Boutry, P. Dudouet, P. Richaud, and R. de Crevoisier, "Benefit of intensity modulated and imageguided radiotherapy in prostate cancer," Cancer Radiothérapie, vol. 14, no. 6-7, pp. 479-487, 2010.

[6] M. J. Zelefsky, M. Kollmeier, B. Cox et al., "Improved clinical outcomes with high-dose image guided radiotherapy compared with non-IGRT for the treatment of clinically localized prostate cancer," International Journal of Radiation Oncology, Biology, Physics, vol. 84, no. 1, pp. 125-129, 2012.

[7] V. Valakh, A. Kirichenko, R. Miller, T. Sunder, L. Miller, and R. Fuhrer, "Combination of IG-IMRT and permanent source prostate brachytherapy in patients with organ-confined prostate cancer: GU and GI toxicity and effect on erectile function," Brachytherapy, vol. 10, no. 3, pp. 195-200, 2011.

[8] M. J. Zelefsky, Y. Yamada, G. N. Cohen et al., "Intraoperative real-time planned conformal prostate brachytherapy: postimplantation dosimetric outcome and clinical implications," Radiotherapy and Oncology, vol. 84, no. 2, pp. 185-189, 2007.

[9] K. M. Snyder, R. G. Stock, S. M. Hong, Y. C. Lo, and N. N. Stone, "Defining the risk of developing grade 2 proctitis following 125I prostate brachytherapy using a rectal dose-volume histogram analysis," International Journal of Radiation Oncology, Biology, Physics, vol. 50, no. 2, pp. 335-341, 2001.

[10] N. Bittner, K. Wallner, G. Merrick, P. Orio, R. Nurani, and L. True, "The time gap between $\mathrm{Pd}-103$ prostate brachytherapy 
and supplemental beam radiation does not impact on rectal morbidity or likelihood of cure," American Journal of Clinical Oncology, vol. 31, no. 3, pp. 231-236, 2008.

[11] T. Sherertz, K. Wallner, G. Merrick et al., "Factors predictive of rectal bleeding after 103Pd and supplemental beam radiation for prostate cancer," Brachytherapy, vol. 3, no. 3, pp. 130-135, 2004.

[12] Y. Shiraishi, A. Yorozu, T. Ohashi et al., "Dose constraint for minimizing grade 2 rectal bleeding following brachytherapy combined with external beam radiotherapy for localized prostate cancer: rectal dose-volume histogram analysis of 457 patients," International Journal of Radiation Oncology, Biology, Physics, vol. 81, no. 3, pp. e127-e133, 2011.

[13] K. Kalakota, E. Rakhno, C. A. Pelizzari, A. B. Jani, and S. L. Liauw, "Late rectal toxicity after prostate brachytherapy: influence of supplemental external beam radiation on dosevolume histogram analysis," Brachytherapy, vol. 9, no. 2, pp. 131136, 2010.

[14] Cancer Therapy Evaluation Program, Common Terminology Criteria for Adverse Events, Version 3.0, DCTD, NCI, NIH, DHHS, March 2003, http://ctep.cancer.gov/.

[15] W. R. Lee, M. DeSilvio, C. Lawton et al., "A phase II study of external beam radiotherapy combined with permanent source brachytherapy for intermediate-risk, clinically localized adenocarcinoma of the prostate: preliminary results of RTOG P-0019," International Journal of Radiation Oncology, Biology, Physics, vol. 64, no. 3, pp. 804-809, 2006.

[16] W. R. Lee, K. Bae, C. Lawton et al., "Late toxicity and biochemical recurrence after external-beam radiotherapy combined with permanent-source prostate brachytherapy: analysis of Radiation Therapy Oncology Group study 0019," Cancer, vol. 109, no. 8, pp. 1506-1512, 2007. 


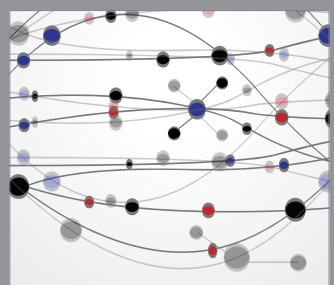

The Scientific World Journal
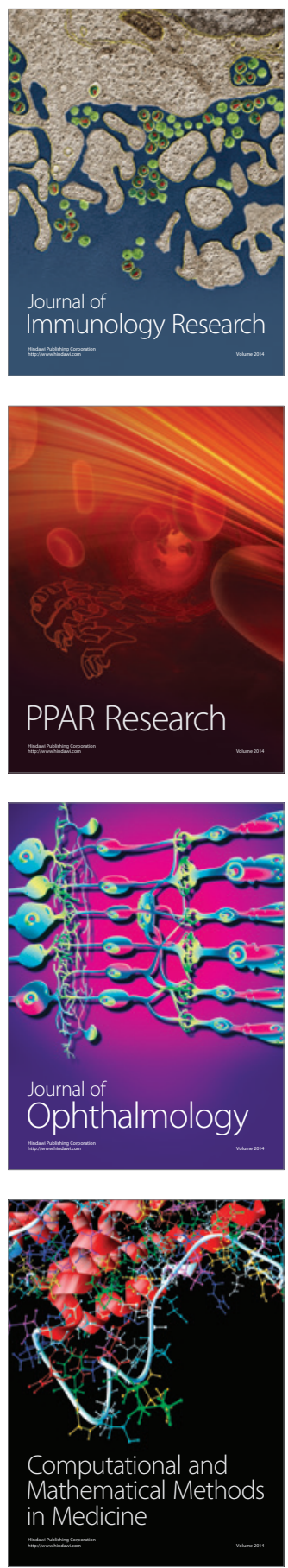

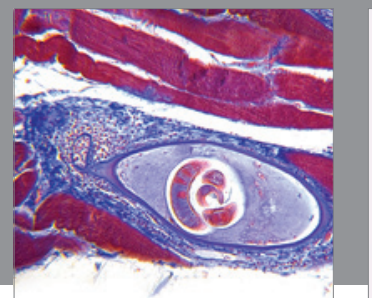

Gastroenterology

Research and Practice
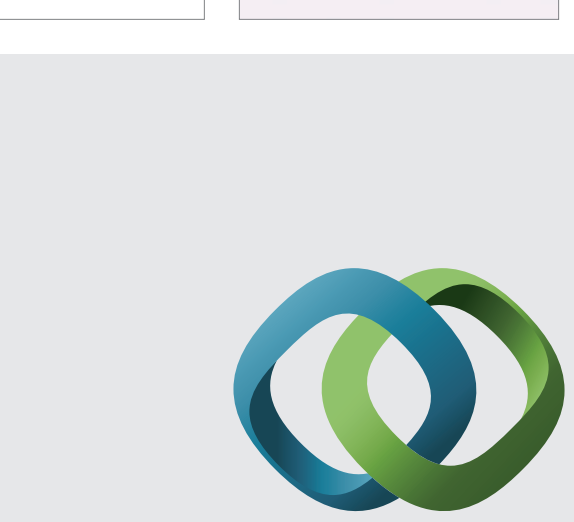

\section{Hindawi}

Submit your manuscripts at

http://www.hindawi.com
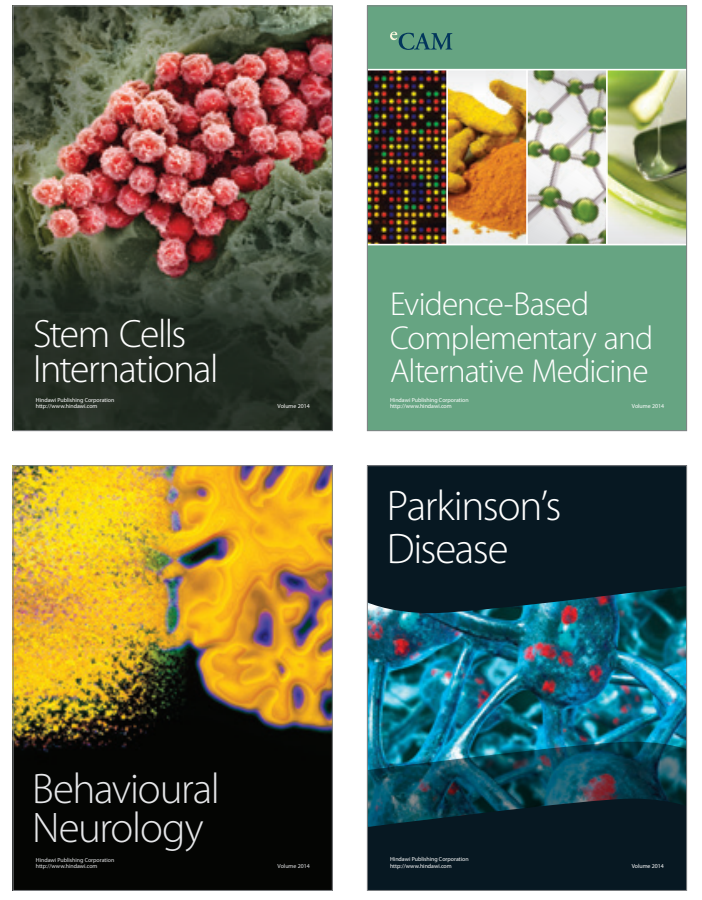
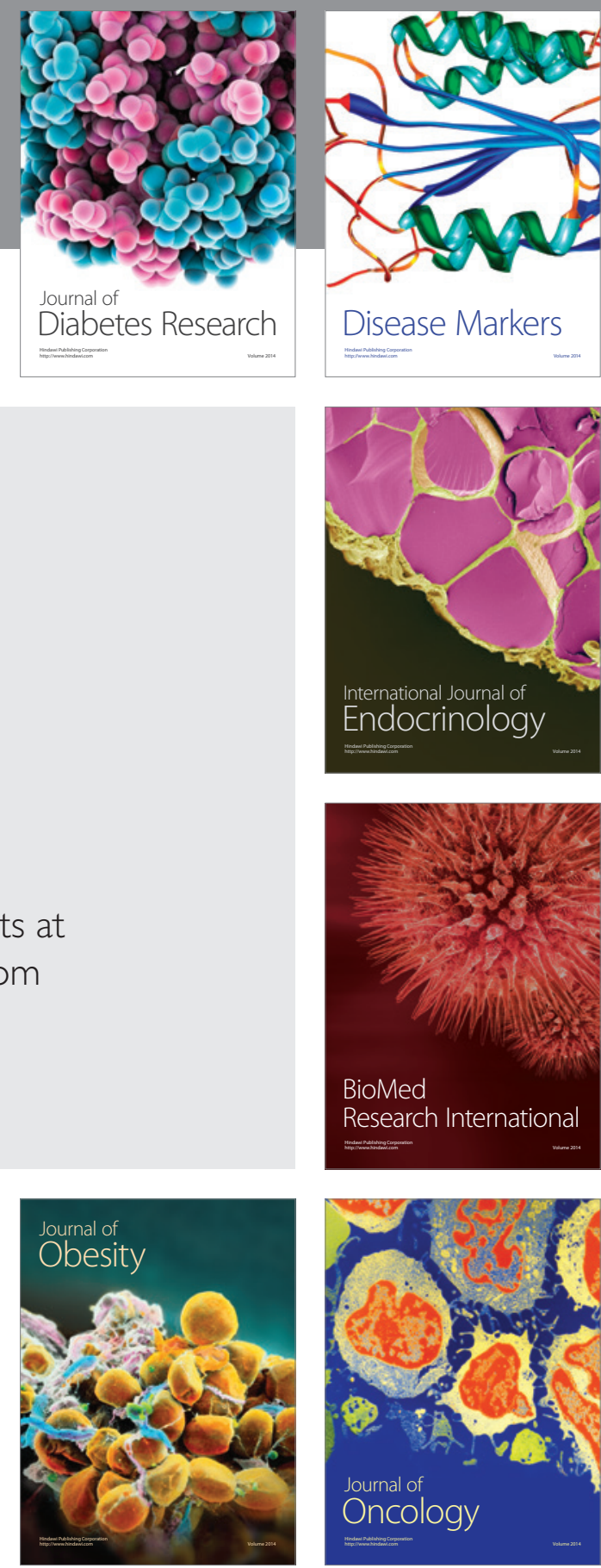

Disease Markers
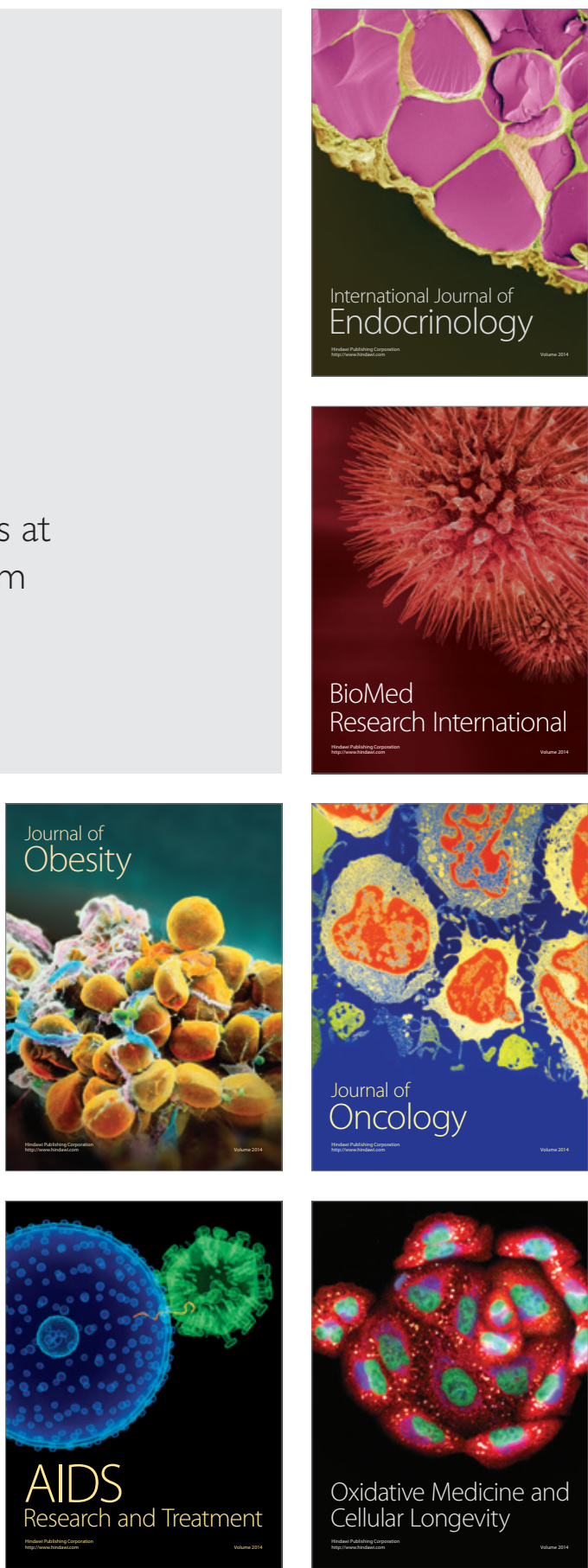